aero-engine to below $7: 1$; hence there is a distinct loss of possible efficiency. Diisobutylene or diamylene, having better anti-knock properties than benzol, could permit of a higher H.U.C.R., and, moreover, blends of these hydrocarbons would not be liable to freeze at high altitudes, both substances being liquid at $-45^{\circ} \mathrm{C}$. in an undiluted state. Diisobutylene may be conveniently prepared by the polymerisation of the isobutylene content of ' cracked' gases by means of sulphuric acid, while diamylene may be obtained by the similar treatment of either trimethyl ethylene or tertiary amyl alcohol.

Since this work was completed it has been found that E.P. 253,131 covers the use of these two olefines, among others, as anti-knock dopes, and describes them as being better than benzol for this purpose, but no comparative figures are quoted.

It is interesting to note that of the olefines we have tested, those which are the more stable towards bromine, sulphuric acid, potassium permanganate, and potassium bichromate, are the more effective in suppressing knocking.

A. W. NASH.

Donald A. Howes.

Department of Oil Engineering and Refining, University of Birmingham.

\section{A Permeability Test with Radioactive Indicators.}

Certain investigators (see, for example, W. J. V. Osterhout, "Some Fundamental Problems in Cellular Physiology," 1927 ; especially pages $36-48$ ) believe that the protoplasm of the living cell is permeable only to undissociated molecules but impermeable to ions.

It seemed possible to me to test this theory with the method of radioactive indicators ${ }^{1}$ (Hevesy-Paneth). The advantage of this method is that only very small amounts of the ions which enter the cell are necessary and that a very small concentration can be detected. Radioactive lead (thorium-B) was used as an indicator for lead ions, and therefore lead nitrate was dissolved in sea water so as to make it $10^{-5}-10^{-6} M$ in respect to lead ions. Cells of Valonia macrophysa were used since the large volume and the amount of sap available make the investigation easier, and since investigations of the permeability of this cell were carried out by Osterhout and his collaborators.

To test whether or not the presence of lead causes any injury to the cell, the cells were placed in sea water with different amounts of lead nitrate added, and for several months the behaviour of the cells observed. The cells did not change in colour or rigidity, and were, according to Dr. L. R. Blinks, who kept them in the same laboratory with other cells, in a normal state, judged from macroscopic appearance.

For the permeability experiments, the cells were placed in sea water containing a known amount of lead nitrate and thorium-B. After 20 or 30 hours the cells were taken out, washed off with inactive sea water, and dried on blotting paper. The sap was removed, a certain amount $(0 \cdot 2-0 \cdot 3$ c.c.) evaporated in a watch glass, and the radioactivity measured in an a-ray electroscope. The activity of the same amount of the original solution and of the sea water in which the cells were kept was measured. In this way we ascertained how much lead is absorbed by the cell wall and how much enters the vacuole. In all experiments (14 cells) it was found that about 50 per cent of the lead ions present in the original solutions are absorbed by the cell wall, but that practically no lead

1 That is, to determine the amount of ions present, of a certain kind, by the determination of the radioactive isotope mixed with them. Since a chemical separation of isotopes is impossible, the change in activity of the radioactive isotopes is the indicator for changes in the concentration of the inactive ion. enters the vacuole. ${ }^{2}$ The same experiments were carried out with cells which had been kept in sea water plus lead nitrate for four months. Also in this case no lead could be found in the vacuole.

One may conclude that all the lead which dis. appears from the sea water is adsorbed by the cell wall or the protoplasm forming an insoluble compound which cannot enter the vacuole. In this case one would expect that in dead cells also the lead would be fixed at the cell walls and therefore cannot be found in the sap. Experiments with three dead cells have shown that lead does enter a dead cell. It is apparently fixed there to small particles of organic matter which are to be found always in dead cells. Therefore it cannot diffuse back into the surrounding sea water and an apparent concentration of lead in the dead cells takes place.

It was interesting to see whether radium emanation, being a rare gas, would enter the cells, as one would expect from the theory. Small capillaries $(16 \mathrm{~mm}$. long), filled with radium emanation (about 0.01 m.c.), were broken under the sea water containing the cells to be tested. It was found that already after one hour the sap is approximately as active as the surrounding sea water ( 15 cells were investigated).

After every experiment, Dr. L. R. Blinks examined the macroscopic appearance of the cells and tested the sap for sulphate ions. (The presence of sulphate ions would indicate a severe injury.) Part of the sap in our lead experiments and the sap of every single cell in the experiments with radium emanation was tested in this way. Injury was found in one cell out of a total of three, exposed for 20 hours in radium emanation, and traces of sulphate ions in two cases out of twelve, after 1 to 2 hours exposure in radium emanation. One cell that had been in lead nitrate for four months was soft, but did not give any sulphate reaction and did not show any sign of injury in our test.

Summary.--Using radioactive indicators for testing the permeability of single cells of Valonia macrophysa, it was found that lead ions do not enter the sap of the living cell even if the cells are kept for several months in lead nitrate solution. Lead ions enter readily the sap of dead cells. Radium emanation, being a rare gas, is already after one hour distributed evenly between the cell sap of living cells and the surrounding sea water.

This investigation was carried out in the spring of 1927 during our stay at the Rockefeller Institute for Medical Research, New York City, and we are indebted to the International Education Board who made our stay at the Rockefeller Institute possible. KARL LARK-HOROVITZ.

Physics Department, Purdue University, Indiana.

\section{Molecular Gonstants of Hydrogen.}

ONE of us recently published a table of constants for the neutral hydrogen molecule (Proc. Nat. Acad. Sci., $14,12 ; 1928)$. The most uncertain quantity in that table was the value of the moment of inertia for the ' $B$ ' level. The value given $\left(1.99 \times 10^{-40}\right)$ is based on Hori's very doubtful interpretation of Witmer's band progression $B_{3}-A_{n}$. We have now photographed the entire $B \cdot A$ system in the second order of a ten-foot vacuum spectrograph, designed by Prof. J. J. Hopfield

2 A trace of activity which was found twice immediately after drying is due to traces of thorium-C. This may have entered the cell in ionic form, but since thorium-C is present only in an extremely small concentration, this is not contradictory to any other experiment on permeability. Such a small amount may possibly also enter in other cases, but could not be detected. On the other hand, thorium-C shows in neutral solutions a quasi-colloidal behaviour and may have entered the cell in form of an undissociated complex. 